\title{
Editorial: As Above So Below? Progress in Understanding the Role of Belowground Interactions in Ecological Processes
}

\author{
Oren Shelef ${ }^{*}$, Philip G. Hahn ${ }^{2}$, Ana Pineda ${ }^{3}$, Mysore V. Tejesvi ${ }^{4,5}$ and \\ Ainhoa Martinez-Medina ${ }^{6}$ \\ ${ }^{1}$ Department of Natural Resources, Institute of Plant Sciences, Agricultural Research Organization (ARO), Volcani Center, \\ Rishon Le Tzion, Israel, ${ }^{2}$ Division of Biological Sciences, University of Montana, Missoula, MT, United States, ${ }^{3}$ Netherlands \\ Institute of Ecology (NIOO-KNAW), Wageningen, Netherlands, ${ }^{4}$ Ecology and Genetics, University of Oulu, Oulu, Finland, \\ ${ }^{5}$ Chain Antimicrobials, Oulu, Finland, ${ }^{6}$ Plant-Microorganism Interaction Unit, Institute of Natural Resources and Agrobiology \\ of Salamanca (IRNASA-CSIC), Salamanca, Spain
}

Keywords: belowground interactions, plant-associated organisms, community ecology, plant-soil continuum, functional ecology

\section{Editorial on the Research Topic}

As Above So Below? Progress in Understanding the Role of Belowground Interactions in Ecological Processes

\section{OPEN ACCESS}

Edited and reviewed by: Jordi Figuerola, Estación Biológica de Doñana (EBD), Spain

*Correspondence: Oren Shelef shelef@volcani.agri.gov.il

Specialty section:

This article was submitted to Behavioral and Evolutionary Ecology,

a section of the journal

Frontiers in Ecology and Evolution

Received: 25 July 2019 Accepted: 07 August 2019 Published: 21 August 2019

Citation:

Shelef O, Hahn PG, Pineda A, Tejesvi MV and Martinez-Medina A (2019) Editorial: As Above So Below? Progress in Understanding the Role of Belowground Interactions in Ecological Processes.

Front. Ecol. Evol. 7:318. doi: 10.3389/fevo.2019.00318
Biotic interactions that occur belowground involve all of the kingdoms of life and can have an immense influence on eco-evolutionary dynamics of the interacting organisms as well as the functioning of ecosystems (Lavelle et al., 2016; Orgiazzi et al., 2016; Coleman et al., 2017). For example, ecosystems are dependent on belowground diversity to regulate nutrient cycles and these interactions provide services critically important for the well-being of the biosphere, including food security, and carbon sequestration (Lal, 2004). Indeed, the importance and complexity of belowground interactions have long been recognized by ecologists and has even permeated into popular culture as illustrated in the quilt art "Mother Earth and Her Children" by Sieglinde Schoen Smith (von Olfers, 2007). While it may not represent a precise objective picture of nature, it does highlight the vital role of belowground interactions. Despite increasing awareness of the importance of belowground interactions and particularly how above- and belowground communities are linked through biotic interactions (Hooper et al., 2000; Wardle et al., 2004; Young and Crawford, 2004), belowground interactions have still received considerably less attention compared to aboveground interactions. This lack of attention is perhaps due to the complexity and many logistical challenges of working belowground (Shelef et al.) and remains a knowledge gap that severely hampers our ability to understand and predict the functioning of terrestrial ecosystems.

Exciting new discoveries published in this Research Topic provide state-of-the-art information on the belowground interactions. The 18 contributions published in this Research Topic explored interactions from a variety of taxonomic groups, such as fungi, bacteria, or invertebrates and include a mix of Mini Reviews, Reviews, Original Research Articles, and a Perspective. Through the development of novel conceptual frameworks and invigorated by modern molecular and chemical techniques, these articles shed new light on how belowground interactions affect ecological processes, from soil health through impacting plant interactions with above-ground fauna. Collectively, this body of work provides substantial progress in our understanding of ecology belowground and linkages with aboveground systems and has the potential to make a lasting 
contribution to the field of ecology. These studies make a significant contribution on several themes in below- and aboveground ecology that are currently "hot topics," such as conceptual approaches to studying interactions belowground, ecological interactions in global change scenarios, patterns, and mechanisms of microbial effects on plant-insect interactions at multiple trophic levels, or factors affecting microbiome assembly belowground.

In a broad ecological context, the article by Shelef et al. highlights the importance of developing a conceptual framework for the study of belowground interactions. The authors indicate that many foundational ecological concepts (e.g., keystone species, island biogeography, trophic cascades) have been developed and tested mainly in aboveground systems and are sparsely referenced in the belowground literature. The authors suggest that while there are some fundamental differences in key ecological processes that occur above- vs. belowground, increased attention on belowground interactions using modern methodological approaches will ultimately help to integrate across the above- and belowground realms. Some of those methodological approaches to studying belowground microbial communities are thoroughly reviewed in the study of Mecado-Blanco et al. Here the authors highlight the importance of belowground microbes in forested ecosystems, as well as their role in mediating ecological processes, and how they influence the productivity of tree crops. They propose a strategy for deploying microbiota to combat not only belowground pests but also abiotic stressors that threaten tree crop production.

Abiotic stress can alter many fundamental ecological interactions and also is one of the predicted consequences of global change, the latter of which is a major societal concern. Hahn et al. examined how short- vs. long-term differences in soil moisture influences the effect of soil microbes on plant functional traits. While there were substantial differences in mycorrhizae communities found under spatially separated sites in the field, these different source communities did not differ in their effects on plant growth. Instead, plant growth responses to mycorrhizae were affected by short-term changes in soil moisture, where plant responses were positive under drought conditions but adverse under wet conditions. Focusing on other abiotic stresses that are predicted to increase in future climate scenarios, Johnson et al. show how silicon supplementation to the soil can maintain plant productivity of Medico sativa under conditions of elevated $\mathrm{CO}_{2}$ and temperature, associated with an increase of root nodulation. This study shows how understanding the belowground interaction between microbes and plants can contribute to ameliorating the predicted impacts of climate change.

Other significant consequences of global change are the colonization of new areas by invasive or range expander species. Toward this end, Liu et al. examined how invasion by a non-native plant influences the composition of soil bacterial communities in wetlands. Using multivariate analyses of $16 \mathrm{~S}$ rRNA gene sequences, they showed that plant invasion alters soil bacterial communities. Interestingly, plant invasion increased species richness but had more nuanced effects on community composition and functional diversity (Liu et al.). At another level, it is still unknown what mechanisms influence host preference for belowground organisms. In the context of species whose distribution change due to climate change, this question is of high importance to predict whether the native herbivores will attack a plant with a novel chemistry. Wilschut et al. address this question, by comparing pairs of three plant species, one range expander and a native one, in terms of volatile emissions and attractiveness to nematodes. Interestingly, the two pairs with the most distinct root volatile profiles between native and range expander, were also where nematodes preferred the native species. Climate change is imposing a plethora of new challenges that can only be faced when we understand the mechanisms by which plants interact with their new pests.

Plant phytohormones, such as jasmonic acid (JA) and salicylic acid (SA) orchestrate induced plant defenses to herbivores and pathogens. Nevertheless, how plants integrate their induced responses under multiple belowground and aboveground interactions remains obscure. Bakhtiari et al. explored how elicitation of root defenses by application of exogenous JA impacts shoot defenses and the resistance against aboveground herbivory. They found that the induction of the JA-pathway in roots altered the abundance and diversity of glucosinolates in the shoots, increasing the plant resistance against aboveground herbivory. The hormonal network regulating abovegroundbelowground interactions seems to be highly influenced by the entity of the attacking organisms. Various paths of hormonal signaling may have a different regulating effect on the response to belowground nematodes. Van Dam et al. found that aphid infestation on plants previously infested with the cyst nematode Heterodera schachtii induced the SA-regulated pathway in aboveground tissues while the JA-regulated pathway was repressed. By contrast, aphid infestation on plants previously infested with the root knot nematode Meloidogyne hapla triggered the JA-regulated pathway. Interestingly, aphids performed worse in plants infested with the cyst nematode, while they performed better in plants infested with the root-knot nematode indicating impact of feeding strategy of the belowground attacker on the aboveground herbivores.

In addition to influencing plant growth, soil microbes can also affect insect herbivores aboveground through changes in plant chemistry. Bernaola et al. showed that inoculation with mycorrhizal fungi improved the growth of rice plants, but also made them more susceptible to insect and fungal pathogen pests. The authors found no difference in nutritional status between inoculated and uninoculated rice plants, suggesting that plant defenses may be responsible for differences in pest performance (Bernaola et al.). Meier and Hunter found that mycorrhizal fungi can increase levels of plant toxins (cardenolides) in several species of the genus Asclepias (Meier and Hunter). Interestingly, herbivores that fed on mycorrhizae-inoculated plants were able to sequester higher amounts of toxins in their own bodies, which may make them more resistant to their own predators. These studies demonstrate how belowground interactions between plants and soil microbes can have important effects on aboveground interactions between plants and insects, but also highlight the variability in the outcome of these interactions. Heinen et al. also point toward that variability and highlights that 
most of the microbe-plant-insect research has been conducted in a laboratory and controlled environments. Heinen et al. then review and show the significant role of soil organisms in shaping plant-insect interactions in the field, similar to what has been shown in controlled conditions. Arbuscular mycorrhizal fungi (AMF) have both positive effects for specialist and adverse effects on generalist herbivores. Moreover, nematodes negatively affect herbivores. Some soil organisms may be promising agents for improving and protecting crop yields and may also influence community-level aboveground plant diversity, suggesting novel possibilities to recruit soil science to control aboveground communities of herbivores.

Examining community-level effects in more depth, a fascinating topic but still scarce in the literature is the impact of belowground communities on the functioning of multitrophic interactions aboveground. The review by Tao et al. summarizes recent literature on the mechanisms by which belowground mutualistic microbes affect predation and pathogen pressure on herbivores aboveground. They provide an excellent overview of how belowground mutualistic microbes influence predator attraction and foraging efficiency and the quality of the prey. However, certain predator species can also display herbivory feeding patterns, and in experimental work, Garantonakis et al. evaluated how the fungal root endosymbiont Fusarium solani affects the predatory bug Nesidiocoris tenuis, which can cause severe plant damage when prey insects are lacking. They show that damage to the plants by this zoophytophagous insect was reduced on Fusarium-inoculated plants (Garantonakis et al.). These studies nicely demonstrate the importance of including multiple trophic levels to fully understand ecosystem functioning and the ecosystem services of belowground communities.

Recently, more research is exploring how aboveground organisms trigger a series of physiological responses in the plant that systemically affect belowground communities, a direction much less studied than from below- to aboveground. Hoysted et al. demonstrates that aphid herbivory alters the root exudates of potato, with a negative effect on nematode egg hatching. Although sugars seemed to play a role, sugar addition did not recover the egg hatch whereas root exudates of uninfested plants did. Lee et al. show how whitefly herbivory suppresses the root bacterial pathogen Ralstonia sonalacearum in Nicotiana benthamiana. They are identifying two effectors (2G5 and 6A10) present in the whitefly salivary glands. It is fascinating that under attack aboveground, plants have developed defense mechanisms that protect them from belowground pathogens.

Belowground microbiomes also play important roles in terrestrial ecosystems and are one of the most cited fields in

\section{REFERENCES}

Coleman, D. C., Callaham, M. A. Jr., and Crossley, D. A. Jr. (2017). Fundamentals of Soil Ecology. London: Academic press.

Hooper, D. U., Bignell, D. E., Brown, V. K., Brussaard, L., Dangerfield, J. M., Wall, D. H., et al. (2000). Interactions between aboveground and belowground biodiversity in terrestrial ecosystems: patterns, mechanisms, and feedbacks. recent years. In microbiome research, a key issue is which factors drive microbiome community assembly and function. Ourry et al. showed that the presence of herbivore damage can influence the soil microbiome, associated with changes in the chemical composition of root exudates (Ourry et al.). An interesting applied benefit of understanding microbiome assembly is harnessing beneficial microbiomes to increase productivity of economically viable crops, which is still a challenge. Ma et al. applied the concept of plant-soil feedbacks to assess how inoculation of soil microbiomes cultured by 37 wild species of grass, forbs, and legumes, affect the growth of the cut flower chrysanthemum. Chrysanthemum grown in soil inoculated with grass legacies tended to be larger and less susceptible to soil-borne diseases than plants grown in soil cultured by forbs or legumes (Ma et al.), suggesting that we can apply this ecological concept to increase the sustainability of crop production.

\section{CONCLUDING REMARKS}

Soils are one of the most diverse habitats on Earth, with microbiota comprising a large portion of this diversity. One theme emerging from this review is the tremendous variation in soil microbial communities and the outcome of interactions between soil microbes and plants. In this Research Topic researchers highlight belowground interactions and outline their myriad ecological roles, from affecting soil health through impacting plant interactions with aboveground fauna. The studies here broadened the conceptual framework for the study of belowground interactions, explored interactions between plants and microbial communities in the soil, and the flow of Plant-mediated interactions between above- and belowground invertebrates. These studies are shedding light on missing details of the belowground complex "Mother Earth and Her Children" is illustrating.

\section{AUTHOR CONTRIBUTIONS}

All authors listed have made a substantial, direct and intellectual contribution to the work, and approved it for publication.

\section{ACKNOWLEDGMENTS}

The ideas for this Research Topic were conceived at a discussion on belowground ecology at the Plant-Herbivore Interactions Gordon Conference in 2017 and we are grateful to the organizers of the conference and participants of these discussion.

Bioscience 50, 1049-1061. doi: 10.1641/0006-3568(2000)050[1049:IBAABB]2.0. $\mathrm{CO} ; 2$

Lal, R. (2004). Soil carbon sequestration impacts on global climate change and food security. Science 304, 1623-1627. doi: 10.1126/science.10 97396

Lavelle, P., Spain, A., Blouin, M., Brown, G., Decaens, T., Grimaldi, M., et al. (2016). Ecosystem engineers in a self-organized soil: a review 
of concepts and future research questions. Soil Sci. 181, 91-109. doi: $10.1097 /$ SS.0000000000000155

Orgiazzi, A., Bardgett, R. D., and Barrios, E. (eds.). (2016). Global Soil Biodiversity Atlas. Luxemburg: European Commission.

von Olfers, S. (2007). "Mother earth and her children: a quilted fairy tale," eds P. J. Zipes and I. S. Schoen-Smith (Elmhurst, IL: Breckling Press), 32 .

Wardle, D. A., Bardgett, R. D., Klironomos, J. N., Setala, H., Van Der Putten, W. H., and Wall, D. H. (2004). Ecological linkages between aboveground and belowground biota. Science 304, 1629-1633. doi: 10.1126/science. 1094875

Young, I. M., and Crawford, J. W. (2004). Interactions and self-organization in the soil-microbe complex. Science 304, 1634-1637. doi: 10.1126/science. 1097394
Conflict of Interest Statement: MT was employed by company Chain Antimicrobials, Oulu, Finland.

The remaining authors declare that the research was conducted in the absence of any commercial or financial relationships that could be construed as a potential conflict of interest.

Copyright $\odot 2019$ Shelef, Hahn, Pineda, Tejesvi and Martinez-Medina. This is an open-access article distributed under the terms of the Creative Commons Attribution License (CC BY). The use, distribution or reproduction in other forums is permitted, provided the original author(s) and the copyright owner(s) are credited and that the original publication in this journal is cited, in accordance with accepted academic practice. No use, distribution or reproduction is permitted which does not comply with these terms. 